\title{
THE RELATIONSHIP BETWEEN USE AND SUITABILITY OF RESOURCES AND ITS CONSEQUENCES TO INSECT POPULATION SIZE
}

\author{
JoRge SOBERón M. \\ Departamento de Ecología, Instituto de Biologia, Universidad Nacional Autonoma de México, \\ Mexico City 04510, Mexico
}

Submitted February 2, 1984; Revised July 3, 1984; Accepted April 3, 1985

The question of what determines population density in the field has always been central to ecology. Sometimes predators and parasites seem to be the main cause of the observed prey densities (Lack 1954; Clark et al. 1967; Beddington et al. 1978; Hassell 1978; Anderson 1979; Lawton and McNeill 1979). Alternatively, the availability of food or other resources has commonly been invoked as the main determinant of population size (e.g., Clark et al. 1967; Pianka 1974; McNaughton and Wolf 1979), resulting in the idea of the "carrying capacity" of an environment. This has been defined as the maximum size that a population can reach in the absence of natural enemies and catastrophes. The interplay between the concepts of carrying capacity and "intrinsic growth rate," as described by the logistic equation, has become the standard general theory of single-species population growth (Slobodkin 1962; Pianka 1974; McNaughton and Wolf 1979).

As in any theoretical model, several simplifying assumptions underlie the logistic equation, many of which have been relaxed and explored by different authors (Wangersky and Cunningham 1957; Roughgarden 1974; Strebel 1980). One of the more fundamental suppositions is that the level of the carrying capacity is set by the availability of resources (Solbrig and Solbrig 1979; Dempster and Pollard 1981; Pollard 1981). This seemingly plausible supposition has seldom been examined critically (see, however, King 1971). It is clear that availability in itself is not necessarily the relevant factor for two reasons. First, the suitabilities of the utilized resources are often heterogeneous; thus, differences in availability do not always reflect differences in suitability. Second, the use that organisms make of their resources is also heterogeneous; thus, some available and suitable resource may remain underutilized or, alternatively, utilized out of proportion to its suitability, an abuse that may lead to heavy mortality (Dethier 1959).

This suggests that it is more relevant to consider the relationship between the patterns of use and the suitability of the different resources than to consider only their availabilities. My purpose here is to discuss this relationship and its consequences for population size. 


\section{THE CONCEPTS OF SUITABILITY AND USE}

The suitability of a food for an animal hinges on the increased fitness derived from using that resource or diet. Suitability, therefore, can be seen as the "sum" of the effects of using a resource on the fecundity and survivorship of the consumers. A natural way of integrating all these effects to quantify suitability is to use the net per capita fecundity

$$
R=\sum_{x} l_{x} m_{x}
$$

where $l_{x}$ represents the probability of surviving from birth to age $x$ and $m_{x}$ is the average number of progeny produced by an individual of age $x$. Thus, for purposes of comparison, one can say that a particular resource $A$ is more suitable than another resource $B$ if $R_{A}>R_{B}$.

Differences in diets can produce differences in the values of $R$. For example, the grasshopper Euthystira brachiptera (Ocksay) was fed three different plant species and three different mixtures (among the hundreds of possible combinations) of these plant species. The mixtures always sustained a higher net fecundity than the single-species diet, and the difference in one case was sevenfold (Kaufmann 1965; see also Chew 1975; Scriber 1979; Mitchell 1981).

Although the idea of measuring the suitability of diets by calculating values of $R$ is appealing, its field measurement in polyphagous species is difficult. In the first place, observing and recording the actual diet for polyphagous individuals (e.g., grasshoppers, rabbits, etc.) may be made difficult by their frequent changes in diet. Second, as mentioned above, each possible diet may have quite different effects on survival rates and fecundity, and the testing of every possible diet and associated value of $R$ would be prohibitively time-consuming.

These difficulties, however, can be minimized by considering species whose individuals are monophagous. This is often the case for holometabolous herbivorous insects, such as butterflies, which may be polyphagous as species, but whose larvae feed mostly on the individual plant upon which the mother laid her eggs. Similarly, many insect parasitoids are polyphagous (or oligophagous) but have monophagous individuals. For such species, the problem of recording the diet reduces to one of finding the location of eggs or larvae. The problem of estimating the suitability of the diet is also lessened, since mixed diets are uncommon. In principle, therefore, one needs only to estimate $R$ values from life tables of several individuals feeding on the different plants. In practice, however, many problems arise. Without even attempting to be exhaustive, I mention some of these problems.

In the first place, such "intrinsic" components of suitability as available nitrogen, secondary components, water content, and toughness are all known to undergo seasonal and ontogenetic cycles (Feeny 1970; Haukioja et al. 1978a,b; Mattson 1980; Dirzo 1984). The microhabitat of a particular plānt can also be a determinant of its quality as food (Singer 1972; Wiklund and Ahrberg 1978) and the same is true for its phenology (Feeny 1970; Holdren and Ehrlich 1982). The 
recent history of a plant can also affect its nutritional quality, as demonstrated by Dolinger et al. (1973), Haukioja and Niemela (1977), and Haukioja (1980).

Second, some components of suitability are related not to food quality per se, but to the associated fauna or flora of a resource. A clear example of this is the association of some plants with herbivore-removing ants (Janzen 1966; Hocking 1975). Many parasitoids are also attracted to the food plants of their hosts (Gilbert 1975; Vinson 1976; Smiley 1978; Lawton and McNeill 1979; Price et al. 1980), and natural enemies should therefore be considered an important component of suitability for herbivorous insects. There are also cases of herbivores gaining benefits from the associated fauna of the food plants, as in many myrmecophilous associations (Hinton 1951; Atsatt 1981).

It is also possible that the physical position of a host plant within the habitat can affect its suitability in ways other than by altering food quality. Rainfall, for example, may be a major cause of early larval mortality in exposed places (Harcourt 1966; Rausher 1979a).

Finally, the density of exploiters can be a component of suitability. A plant likely to be defoliated by high numbers of exploiters is an unsuitable plant, and in some cases, females take this into account before ovipositing ("egg load assessment"; see Prokopy 1972; Rothschild and Schoonhoven 1977; Rausher 1979b). Despite these complications, there is no doubt that one can find and measure patterns of suitability both in the laboratory (Kaufmann 1965; Scriber 1979; Dirzo 1980; Wiklund 1981) and in the field (Chew 1975; Rausher 1979a, 1980; Courtney 1981); thus, although difficult, the study of the relation between use and suitability should be possible.

The use that a population makes of a set of potential food resources depends on the rates of search and encounter with the resources and on the rate of oviposition once the resource is found. I am not concerned with the details of the oviposition process, however. For the purpose of this paper, it is sufficient to consider the pattern of oviposition in the different resources as given, stressing the consequences instead of the causes. An important point is that, although oviposition rates are generally proportional to the suitabilities of resources, there are many exceptions to this that warrant some discussion.

In the first place, as already discussed, the true suitability of a plant can change for many unrelated and unpredictable reasons, often making difficult its correct assessment. Even assuming unchanging suitabilities, an insect can still fail to make the proper choice because of, for example, confusing cues from background vegetation (Atsatt and O'Dowd 1976; Price et al. 1980). Some physical features of the habitat, such as differences in insolation (Rausher 1979a; Hirose et al. 1980), may also prevent resources from being fully used.

Sometimes perfectly suitable plants can mask themselves from exploiters, as in some species of Passiflora vines that mimic plants not eaten by the butterfly genus Heliconius (Gilbert 1975). In other cases, suitable plants present structures that advertise them as unsuitable, as with the egg mimics reported in certain populations of Passiflora species (Gilbert 1977; Williams and Gilbert 1981) and in the crucifer Streptanthus breweri Graz (Shapiro 1981). These structures significantly decrease the oviposition rates (Shapiro 1981; Williams and Gilbert 1981). 
Finally, it is also possible that unsuitable resources elicit oviposition. The pierid butterflies studied by Chew (1977) laid more than half of their eggs on a plant lethal to early-stage larvae. These oviposition mistakes are probably caused by the recent association of the herbivores with this newly introduced alien plant (Chew 1977; see also Copp and Davenport 1978; Courtney 1981).

These examples highlight the real possibility of mismatch between patterns of use and suitability. My purpose is to explore the consequences, at the population level, of the variation between these patterns. The simple mathematical models that follow will help clarify the relationship between population size and use and suitability, suggesting ways in which this relationship may be measured.

\section{MATHEMATICAL MODELS}

Let us imagine a univoltine insect with generations divided into two parts, the first of duration $h$ and the second $1-h$. In generation $t$, the dispersal, searching, and oviposition behaviors that determine the actual use made of particular resources take place in the interval $t$ to $t+h$. Since resources can be either physically isolated patches of food plants or hosts, or different species of these, the term "element" will henceforth be used for both unless a distinction is necessary. In principle, every element is available to all animals, although in practice many or all elements may be visited by only a few animals. During the second part of the generation, $t+h$ to $t+1$, the effects of the choice of elements are felt. A good choice means that eggs are oviposited in a suitable element which, as mentioned in the introduction, corresponds to high values of $R=\Sigma_{x} l_{x} m_{x}$.

Now, let $\mathbf{U}_{t}$ be the vector of use of the elements by the population of generation $t$. That is, $\mathrm{U}_{t}=\left(U_{1, t}, U_{2, t}, \ldots, U_{m, t}\right)$, where $U_{i, t}$ is the number of eggs laid in element $i$ in generation $t$ (up to the time $t+h$ ) and $m$ is the number of elements. We now define the total number of eggs laid as $U_{t}=\Sigma_{i} U_{i, t}$, such that $u_{i, t}=U_{i, t} / U_{t}$, the proportion of eggs laid in element $i$. A further vector, $\mathbf{u}_{t}=\left(u_{1, t}, u_{2, t}, \ldots, u_{m, t}\right)$, has the obvious property $\sum_{i=1}^{m} u_{i . t}=1$.

If we now assume that each adult lays an average of $F$ eggs during its active life (period $t$ to $t+h$ ), then $U_{t}=F n_{t}$, where $n_{t}$ is the total adult population at the beginning of the generation, and

$$
U_{i, t}=u_{i, t} F n_{t} .
$$

Now let $\phi_{i, t}$ be the fraction of eggs in element $i$ that survive to yield adults of the next generation. Then, in the element $i$,

$$
n_{i, t+1}=U_{i, t} \phi_{i, t}=u_{i, t} F n_{t} \phi_{i, t},
$$

where $n_{i . t+1}$ is the adult population produced by element $i$. The total adult population $n_{t+1}$ is obtained by summing over all elements:

$$
n_{t+1}=\sum_{i=1}^{m} n_{i, t+1}=F n_{t} \sum_{i=1}^{m} u_{i, t} \phi_{i, t} .
$$

The survival functions $\phi_{i, t}$ depend on the density of exploiters $\left(U_{i, t}\right)$ and on the suitability of the element. The values of $\phi_{i, t}$ can be interpreted as the probabilities 
of reaching the adult age under the given choices of element and density of exploiters. For an insect life cycle, these values correspond to the last value of $l_{x}$ in the equation for the net fecundity $R=\Sigma_{x} l_{x} m_{x}$. Equation (3) thus includes the effects of suitability on survival rates. To do the same for fecundity would require an additional equation that would complicate the analysis. This will not be attempted here.

With the above conventions in mind, equation (3) represents the growth of a population in terms of the average fecundity, the fractional use of each environmental element, and the fractional survival, which has both density-independent (DI) and density-dependent (DD) components. We now turn to the simplest case.

\section{Suitability Affects Only Density-Dependent Mortality}

It is assumed here that $F$ represents only that fraction of eggs that suffers DD mortality. $F$ thus represents the "effective fecundity" of Bellows (1981) and Waage and Hassell (1982). At the same time, DI mortality is assumed to operate equally on all elements, making $\phi_{i, t}=\phi_{i, t}\left(u_{i} F n_{t}\right)$. Later, differences in DI mortality between the elements will also be considered.

To ensure the existence of an equilibrium, $n^{*}$, in equation (3), it is required that $\mathbf{u}$ does not change over time. The case of variable $\mathbf{u}$ is discussed in Soberon (1982). Now, if the functions $\phi_{i, t}\left(n_{t}\right)$ are differentiable and $d \phi_{i, t} / d n_{t}<0$ for all reasonable values of $n_{t}$, there is a single $n^{*}$ such that $n_{t+1}=n_{t}=n^{*}$, and this is the sole equilibrium point of equation (3). This follows because a sum of decreasing functions (such as $\phi_{i, t}$ ) is also decreasing, and thus a graph of $\sum_{i=1}^{m} u_{i} \phi_{i}\left(n_{t}\right)$ versus $n_{t}$ crosses the horizontal line at $1 / F$ only once. From equation (3) wa find that $n^{*}$ must satisfy

$$
1=F \sum_{i=1}^{m} u_{i} \phi_{i}\left(u_{i} F n^{*}\right)
$$

The problem is to obtain from equation (4) an explicit solution $n^{*}=n^{*}(\mathbf{u}, F, \mathbf{S})$, where $\mathbf{S}$ is the vector of parameters defining the suitabilities of the elements (i.e., defining the shapes of $\phi_{i}$ ). Given such a solution, it would be possible to explore how patterns of use could affect population sizes. In particular, it is interesting to find the pattern of use that maximizes $n^{*}$. The problem of maximizing $n^{*}$ can be solved in general (see the Appendix), provided that an explicit solution for equation (4) exists. The only interesting cases in which the solution is known rely on linear survival functions. For example, let $S_{i}$, which represents the suitability of element $i$, be high in those elements in which the effect of density on mortality is low, and vice versa. It is then reasonable to make

$$
\phi_{i}=1-u_{i} F n_{t} / S_{i},
$$

which is the feedback term of a discrete logistic equation (Maynard Smith 1968). Equation (5) has the drawback of permitting negative (i.e., nonsensical) values of $\phi_{i}$. This can happen only when the population is fluctuating around an unstable equilibrium at high values of $F$, and thus it will not affect the present argument. 
Substituting equation (5) into equation (3) gives

$$
n_{t+1}=F n_{t}\left(1-F n_{t} \sum_{i=1}^{m} u_{i}^{2} / S_{i}\right),
$$

which describes the population growth of the whole system assuming linear perelement mortality. We also define the relative suitability of element $i$ as $s_{i}=S_{i} / S$, with $S=\Sigma_{i=1}^{m} S_{i}$ as a measure of the total suitability of the system of elements. Rearranging equation (6) and defining

$$
C_{\mathrm{M}}=\left(\sum_{i=1}^{m} u_{i}^{2} / s_{i}\right)^{-1}
$$

finally yields

$$
n^{*}=S(F-1) C_{\mathrm{M}} / F^{2} \text {. }
$$

Since the equilibrium population for a homogeneous environment ( $m=$ number of elements $=1)$ is simply $n^{*}=S(F-1) / F^{2}$, it is clear that the heterogeneity of use affects the population size via the parameter $C_{\mathrm{M}}$.

It is possible to show, by maximizing $C_{\mathrm{M}}$ subject to $\sum_{i=1}^{m} u_{i}=1$ (and see Hurlbert 1978), that $s_{\min } \leq C_{\mathrm{M}} \leq 1$. The parameter $C_{\mathrm{M}}$ takes its minimum value $s_{\min }$ when $u_{\min }=1$ and $u_{i}=0$ for all the other elements. This hardly surprising result means that the equilibrium size (eq. 8) is minimal when the population uses only the least suitable element. On the other hand, $C_{M}=1$ only when $u_{i}=s_{i}$ for all elements; that is, the population is largest when every element is used in direct proportion to its suitability. This result and some of its implications will be considered more fully later. Meanwhile, the biological meaning of $C_{\mathrm{M}}$ will be discussed in some detail.

\section{Biological Meaning of ' $C_{\mathrm{M}}$ '}

Since $C_{M}$ is at a maximum when elements are exploited in proportion to their suitabilities, it is tempting to interpret it as a measure of grain response (MacArthur and Levins 1964; MacArthur 1968). As a matter of fact, MacArthur did propose an index of "departure from fine grainedness," which is closely related to $C_{M}$ (hence the subscript).

More recently, ecologists (Wiens 1976) defined the response of a population to a mosaic of resources as fine- or coarse-grained depending on the use the population makes of the resources in relation to their abundances. This is close to the original idea of MacArthur and Levins (1964). In this paper, "abundance" is replaced by " "suitability," and therefore a new term is needed. The degree of association between the pattern of use that a population makes of its resources and the pattern of suitabilities of these is called the "euchresis" of the population (see fig. 1). The term was coined using the Greek roots for use and benefit.

For this definition, "degree of association" is taken as degree of fit between the two vectors $\mathbf{u}$ and $\mathbf{s}$. A total euchresis then corresponds to a perfect fit between $\mathbf{u}$ and $\mathbf{s}$ and a partial euchresis to a bad fit. The index $C_{\mathrm{M}}=1 /\left(\sum_{i=1}^{m} u_{i}^{2} / s_{i}\right)$ is related to 


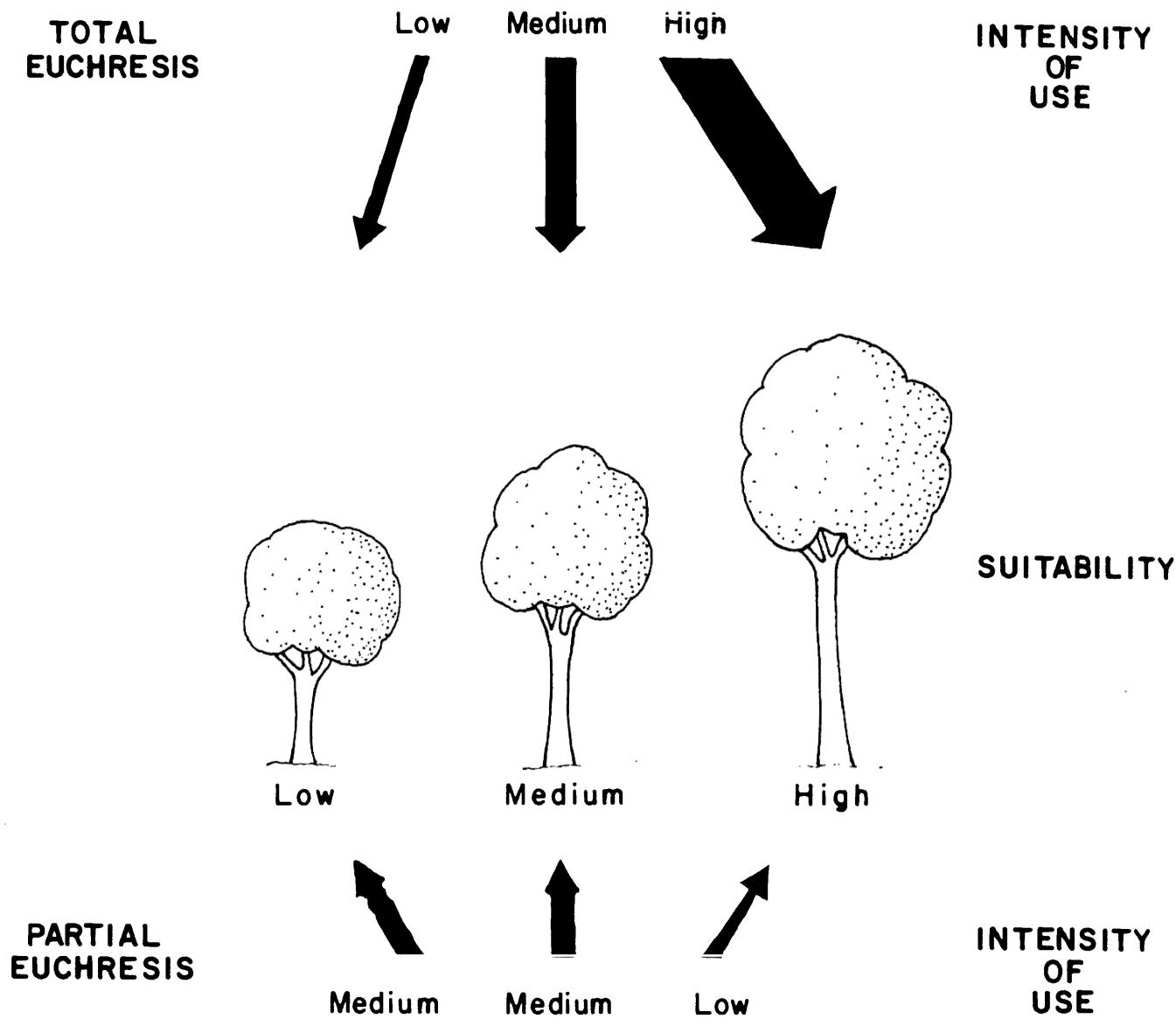

FIG. 1.-A graphic representation of the idea of euchresis. The pattern of use in the top fits the pattern of suitability (represented by the size of the trees), and this corresponds to total euchresis. Conversely, the pattern of use in the bottom does not fit the suitability leading to partial euchresis.

a $\chi^{2}$ goodness of fit (MacArthur 1968; Petraitis 1981), but alternative measures could be used equally well. The results of using other measures of euchresis are mentioned below. It is important to stress that euchresis is considered a property of populations, not of single individuals. Although it may be useful in certain contexts to speak of the euchresis of an individual, for our purposes it is better to avoid any ambiguity and restrict the use of the concept to whole populations and only to those elements that are at least potentially available to every member of the population.

Euchresis may be thought of as the distribution of a population in relation to the potential adult production per element. Overexploitation of the good elements leads to heavy DD mortality, and hence fewer than the maximum possible adults are produced. Too few users implies a wastage of the element and hence also a smaller production of adults. 
Clearly, then, equation (8) implies that population size should be a direct function of the euchresis of the population. Equation (8) also suggests defining the carrying capacity, $K$, as the population equilibrium under perfect euchresis $\left(C_{\mathrm{M}}=\right.$ 1 , thus $\left.K=S(F-1) / F^{2}\right)$. This definition is in accord with the definition of carrying capacity as the maximum number of animals that the environment can support given constant total fecundity and total suitability. However, just by changing the pattern of use (basically a behavioral parameter), a population can be in equilibrium below its theoretical maximum $\left(n^{*}<K\right)$. The euchresis (as measured by $C_{\mathrm{M}}$ ) now emerges as the equilibrium population size, $n^{*}$, relative to the carrying capacity $C_{\mathrm{M}}=n^{*} / K$, and can be seen as a measure of by how much an inadequate oviposition behavior prevents the carrying capacity from being reached. This is in certain ways analogous to the $q=n^{*} / K$ value proposed by Beddington et al. (1978), which measures the impact of natural enemies on the carrying capacity of their prey (defined as the equilibrium population in the absence of enemies).

Populations with partial euchresis may be in an equilibrium well below their carrying capacities. Possible causes of partial euchresis have been mentioned in the section on use and suitability.

\section{Suitability Affects Both Density-Dependent and Density-Independent Mortalities}

In order to introduce DI mortality in equation (3), we need to distinguish between two extreme cases: a population in which DI mortality acts first, followed by a DD mortality; and the opposite situation, in which DD mortality operates before DI mortality. This distinction is similar to the one made by May et al. (1981) about the relative position of parasitism and any additional DD mortality in the host's life cycle. Using the linear equation of Maynard Smith (1968), it is easy to write an expression for $\phi_{i}$. If the fraction of the population $1-D_{i}$ is killed before DD mortality occurs, then only $u_{i} F n_{t} D_{i}$ individuals survive to compete, and we obtain

$$
\phi_{i}=D_{i}\left(1-u_{i} F n_{t} D_{i} / S_{i}\right) .
$$

If, on the other hand, DI mortality occurs after DD, the correct equation becomes

$$
\phi_{i}=D_{i}\left(1-u_{i} F n_{t} / S_{i}\right) .
$$

The corresponding equilibria are

$$
n^{*}=\left[S /(F D)^{2}\right]\left(F D \Sigma u_{i} d_{i}-1\right) /\left(\sum u_{i}^{2} d_{i}^{2} / s_{i}\right)
$$

and

$$
n^{*}=\left[S /\left(F^{2} D\right)\right]\left(F D \Sigma u_{i} d_{i}-1\right) /\left(\Sigma u_{i}^{2} d_{i} / s_{i}\right),
$$

with $D=\sum_{i=1}^{m} D_{i}$ and $d_{i}=D_{i} / D$.

The DD feedback term is now modified by the presence of the proportional survival coefficients $\left(d_{i}\right)$. The control term in each model differs according to the time of action of the DI mortality. In the single-parameter equation (5), suitability 
was equated with the parameter $S_{i}$ of the DD survival functions. By introducing the coefficients $D_{i}$, we have not only another parameter related to suitability, but also the relative timing of the two components affecting survival (the DD and DI mortalities). Thus, to define the suitability of a given element, one has to know (or to postulate a model of) the precise relationship between adult production and the intensity of use in the element in question. It is still possible to regard those elements with higher adult production as the most suitable ones, but it is impossible to quantify the idea without making reference to some explicit model of population survival. Such a model generally has several parameters. We can retain the original definition of euchresis as the degree of association between use and suitability, but it becomes impossible to identify "association" with "goodness of fit" in a strict sense because suitabilities cannot in general be represented by a single number. By the same token, other measures of euchresis may be more complicated than $C_{\mathrm{M}}$ and will also be model-dependent.

What can be used as a measure of euchresis in these more complicated situations? In the preceding section, it was shown that $n^{*} / K=C_{\mathrm{M}}$, where $K$ was the population equilibrium evaluated at maximum $C_{\mathrm{M}}$. This approach can be reversed and the quotient $n^{*} / K$ chosen as the measure of euchresis, with $K$ as the maximum value of $n^{*}$ with respect to the use vector $\mathbf{u}$. To do this we need to maximize $n^{*}$ as defined in equations (11) and (12) subject to $\sum_{i=1}^{m} u_{i}=1$. The method of finding extreme values of $n^{*}$ and the ensuing derivation of an explicit formula for $K$ for the two models (9) and (10) is outlined in the Appendix. The measures of euchresis obtained by this procedure are denoted generically by $C_{\text {fit }}$ :

$$
\begin{aligned}
& C_{\text {fit }}=\frac{1}{C_{\max 1}} \cdot \frac{F D \sum u_{i} d_{i}-1}{\sum u_{i}^{2} d_{i}^{2} / s_{i}}=\frac{C_{1}}{C_{\max 1}} \\
& C_{\text {fit }}=\frac{1}{C_{\max 2}} \cdot \frac{F D \sum u_{i} d_{i}-1}{\sum u_{i}^{2} d_{i} / s_{i}}=\frac{C_{2}}{C_{\max 2}}
\end{aligned}
$$

$C_{1}$ and $C_{2}$ measure the match between actual use and suitability, and $C_{\max 1}$ and $C_{\max 2}$ are the maximum possible values of each match. These quantities are defined in the Appendix. Both equations (13) and (14) collapse to $C_{\mathrm{M}}$ when differences in DI survival are unimportant among patches $\left(D_{i}\right.$ constant for all $\left.i\right)$.

Any particular euchresis index is exact, therefore, only for the model for which it was obtained, but it might be an approximate measure for other models. A true measure of euchresis for a given model should be strictly proportional to $n^{*}$.

To explore the consequences of using nonlinear models of survival $\left(\phi_{i}\right)$, numerical simulations were performed. For given suitability parameters and model $\phi_{i}$, random-use (u) vectors were generated, and several euchresis indexes and values of $n^{*}$ (obtained by iterating the original difference equations) were calculated. Regressions between $n^{*}$ and the euchresis indexes were all significantly positive (Soberón 1982). $C_{\mathrm{M}}$ and $C_{\mathrm{fit}}$ were tested, as well as an index of "weighted niche breadth" proposed by Petraitis (1979), which is closely correlated to $n^{*}$ (only DD mortality). This index, defined as $C_{\mathrm{P}}=\exp \left[\Sigma_{i=1}^{m} u_{i} \ln \left(s_{i} / u_{i}\right)\right]$, has convenient statistical properties (Petraitis 1979, 1981) and may be used instead of $C_{\mathrm{M}}$. 
In the models in which suitability was represented by a single parameter vector (S), the maximum possible value of a euchresis measure was one. In the new models of this section, $C_{\max }\left(C_{\max 1}\right.$ or $\left.C_{\max 2}\right)$ is a variable, and hence the value of $C_{\text {fit }}$ can decrease or increase because of changes in the carrying capacity (defined by $C_{\max }$ ). Whenever $C_{\max }$ changes, the proper correlate of population size will be $C_{1}$ (or $C_{2}$ ) rather than $C_{\text {fit }}$. Although more complicated, this is not different in essence from what was described for single-parameter functions. Euchresis can still be regarded as a relative measure of the utilization of suitability with $C_{1}$ (or $C_{2}$ ) being the actual, realized use of suitability and $C_{\max }$ the maximum possible use, given patterns of $\mathbf{S}, \mathbf{D}$, and fixed fecundity $F$ (D is the vector of DI suitability parameters, $\left.D_{i}\right)$.

Although the conclusion of this section is that, strictly speaking, there should be a euchresis measure appropriate for each particular case, in practice only a few are likely to be used, such as those derived from linear models and in particular $C_{\mathrm{M}}$ and $C_{\text {fit }}$. These are simple and intuitive and have statistical properties that are relatively easy to obtain. On the other hand, $C_{\mathrm{M}}$ and $C_{\mathrm{P}}$ should be good approximations whenever the $D_{i}$ 's are roughly constant and the effective fecundity $(F D / m)$ is small, since small fecundities lead to better fits of $n^{*}$ versus $C_{\mathrm{M}}$ or $C_{\mathrm{P}}$ in the nonlinear models (Soberón 1982).

\section{EMPIRICAL RESULTS}

The basic hypothesis presented here is that there should be a direct relationship between population size and the degree of association between the use and the suitability of resources (euchresis). Several technical assumptions are required in order to derive the hypothesis mathematically (i.e., linearity and discrete generations); I propose, however, that the central biological assumption is that the resources are not affected dynamically by the consumers. This is not as restrictive as it may appear, since it is often the case that herbivore numbers change more easily as a result of variations in plant numbers than vice versa (Crawley 1983). Testing the hypothesis of a relation between euchresis and population size is difficult because of the extensive and detailed information required to quantify use, suitability, and population size. Few published works provide this kind of comprehensive data, but for lack of an ad hoc study, one of these examples will be analyzed to illustrate the ideas presented in the earlier sections.

But first, the methods of testing statistical hypotheses about euchresis indexes deserve some mention. By assuming total euchresis and sampling errors only in the $u$ 's, it can be shown that $U\left(1 / C_{\mathrm{M}}-1\right)$ and $-2 U \ln C_{\mathrm{P}}$ are approximately distributed as $\chi^{2}$ variables with $m-1$ degrees of freedom (Petraitis 1979, 1981). This allows one to obtain the density distributions of sampling, $C_{\mathrm{M}}$ and $C_{\mathrm{P}}$ (Soberón 1982), and the testing of two null hypotheses: total euchresis $\left(C_{\mathrm{M}}\right.$ [or $\left.C_{\mathrm{P}}\right]$ $=1)$ and equality between two measures of euchresis $\left(C_{\mathrm{M}}=C_{\mathrm{M}}^{\prime}\right.$ [or $\left.\left.C_{\mathrm{P}}=C_{\mathrm{P}}^{\prime}\right]\right)$. Whenever the value of the euchresis index is very different from one or when more-complicated indexes are used (e.g., $C_{1}$ or $C_{2}$ ), Monte Carlo simulations can be used to obtain standard errors for the indexes. (For further discussion of this 
topic, see Ricklefs and Lau 1980; Smith 1982; for a concrete application to euchresis, see Soberón 1982.)

In a detailed study on the population dynamics of the swallowtail Papilio xuthus L. in a suburban area in Japan (Hirose et al. 1980), life tables were obtained for different densities of the butterfly in four citrus groves with differing mortalities. Data on the use of the plots and on the total number of adults were also supplied. The authors identified four generations per year and provided 16 life tables, four per year per grove. The only apparent DD mortality acting in all the groves was egg parasitism by Trichogramma wasps. The remaining mortality was in most cases DI but differed from grove to grove. The data suggest that a linear model for $\phi_{i}$, as in equation (10), would be appropriate, namely, $\phi_{i}=D_{i}\left(1-u_{i} F n_{t} / S_{i}\right)$, where $u_{i} F n_{t}$ is the number of eggs laid in grove $i$ at the beginning of generation $t ; 1 /$ $S_{i}$ is the slope of the regression in grove $i$ of survival to first instar versus eggs laid; and $D_{i}$ is the fraction surviving from larvae to adults. The regressions were significant in all but grove $\mathrm{D}$, but this is also included because all four values of $S_{i}$ are needed to calculate euchresis. The data used to calculate the index $C_{\text {fit } 2}$ (eq. 14) appear in table 1 . Notice that although there is one vector $\mathbf{d}$ for each generation, the vector $\mathbf{s}$ is common to all generations (data of the four generations are needed to obtain s). During the second generation the survival was zero in grove $\mathbf{C}$, and since it is not possible to have zeros in either $\mathbf{S}$ or $\mathbf{D}$ because the numbers are used as denominators, the corresponding value of $D$ was arbitrarily set to the average value of $D$ in grove $C$ divided by 10 .

The euchresis values are shown in table 2 . The standard errors were obtained by the Monte Carlo method. Some 200 sets of vectors $\mathbf{s}, \mathbf{d}$, and $\mathbf{u}$ were generated with probabilities taken from the corresponding vectors in table 1 . These "sampled" vectors, $\mathbf{s}, \mathbf{d}$, and $\mathbf{u}$, were then used to calculate the "sampled" $C_{\text {fit }}$, and from these the standard errors were obtained. (For details of the procedure, see Soberón 1982. The formulas for obtaining $C_{\max 2}$ are given in the Appendix.) Table 2 clearly indicates partial euchresis resulting from the overutilization of groves $\mathrm{A}$ and $\mathrm{B}$, which, although less suitable to eggs and larvae (they have smaller values of $S_{i}$ and $D_{i}$ ), are more sunny and hence more attractive to the butterflies. The euchresis figures suggest that the butterfly population is underutilizing its environment by nearly $50 \%$. Of course, this assumes that adult survival is the same in shaded and sunny. groves.

Making use of equation (A8) for the optimal utilization vector $\left(\mathbf{u}_{\text {opt }}\right.$ ), it is possible to predict that simply by displacing $50 \%$ of the eggs in groves $\mathrm{A}$ and $\mathrm{B}$ to grove $C$ (and thus altering $u$ ), the population would increase by about $60 \%$. This increase would occur only because of the difference in the pattern of use, since neither S, D, nor $F$ is assumed to change. Notice, however, that since the DD mortality is caused by parasitism, changes in $\mathbf{u}$ are likely to produce changes in $\mathbf{S}$, perhaps after some delay. Table 2 shows that euchresis in the swallowtails does not differ significantly from generation to generation. This means that if the population is at equilibrium, the realized fraction of carrying capacity $\left(n^{*} / K\right)$ is the same in all generations.

It is possible to use the values of $C_{\max 2}$ to estimate $K$ of the four groves. For the model assumed (eq. 10), the carrying capacity $K=S C_{\max 2} / F^{2} D$. The values of 
TABLE 1

Use and Suitability Data of $P$. xuthus (from Hirose et al. 1980)

\begin{tabular}{|c|c|c|}
\hline Generation & Use and Suitability Vectors & $\begin{array}{c}\text { Total Density-Independent } \\
\text { Suitability }\end{array}$ \\
\hline 1 & $\begin{array}{l}\mathbf{u}=(.369, .433, .114, .084) \\
\mathbf{d}=(.076, .085, .114, .725)\end{array}$ & $D=.236$ \\
\hline II & $\begin{array}{l}\mathbf{u}=(.327, .261, .266, .145) \\
\mathbf{d}=(.061, .370, .013, .606)\end{array}$ & $D=.231$ \\
\hline III & $\begin{array}{l}\mathbf{u}=(.387, .314, .200, .099) \\
\mathbf{d}=(.161, .247, .046, .546)\end{array}$ & $D=.542$ \\
\hline IV & $\begin{array}{l}\mathbf{u}=(.512, .292, .122, .073) \\
\mathbf{d}=(.155, .167, .253, .425)\end{array}$ & $D=.221$ \\
\hline \multirow[t]{2}{*}{. Overall } & $\begin{array}{l}\mathbf{u}=(.398, .325, .180, .100) \\
\mathbf{d}=(.124, .215, .088, .571)\end{array}$ & $D=.307$ \\
\hline & $\begin{aligned} \mathbf{S} & =(32.8,15.87,45.45,40) \\
\mathbf{S} & =(.245, .118, .339, .298) \\
\mathbf{u}_{\text {opt }} & =(.307, .083, .539, .08)\end{aligned}$ & \\
\hline
\end{tabular}

NoTE. - $\mathbf{u}$ values are vectors of proportion of use.

d values are vectors of proportion of density-independent suitabilities.

$\mathbf{S}$ is the vector of density-dependent suitabilities.

$\mathbf{s}$ is the vector of proportion of density-dependent suitability.

$\mathbf{u}_{\text {opt }}$ is the optimum vector obtained from eq. A8.

$D$ is the total per-generation density-independent suitability (see text for explanation).

TABLE 2

Euchresis Values in a Population of $P$. xuthus

\begin{tabular}{clcc}
\hline \hline & \multicolumn{3}{c}{ Euchresis* } \\
\cline { 2 - 4 } GENERATION & $C_{\text {fit }}(\mathrm{SE})$ & $C(\mathrm{SE})$ & $C_{\max }(\mathrm{SE})$ \\
\hline I & $.494(.1)$ & $11.32(2.37)$ & $23.69(.757)$ \\
II & $.536(.1)$ & $13.92(2.4)$ & $26.28(2.01)$ \\
III & $.496(.082)$ & $30.15(4.78)$ & $62.31(3.76)$ \\
IV & $.556(.067)$ & $10.17(1.23)$ & $18.93(.29)$ \\
\hline
\end{tabular}

* $C_{\text {fit }}$ obtained from eqs. A5-A7. Standard errors (SE) obtained by Monte Carlo simulations.

$C_{\max 2}$ appear in table 2, and data in Hirose et al. (1980) yield the values of $S=$ 134.12 and $D=.31$ per square meter and $F=200$ (average number of eggs per adult). The average value of $K$ is thus 94 adults $(.35$ adults $/ \mathrm{m} \times 264 \mathrm{~m})$. The average equilibrium, $n^{*}$, should therefore be about 50 adults (because $C_{\mathrm{fit}}$ is about 0.5). The studies of Hirose et al. (1980) arrived at an average number of adults of $53 \pm 20(\mathrm{SE})$. Although the predicted value is within the error margin, our $K$ value is an underestimation because there were other nonsampled groves in the area, making the theoretical average $\left(n^{*}\right)$ also an underestimation.

Notice that the value of $C_{\max 2}$ changes significantly every generation. Under these conditions, one would expect $C_{2}$ rather than $C_{\text {fit } 2}$ to be correlated with 


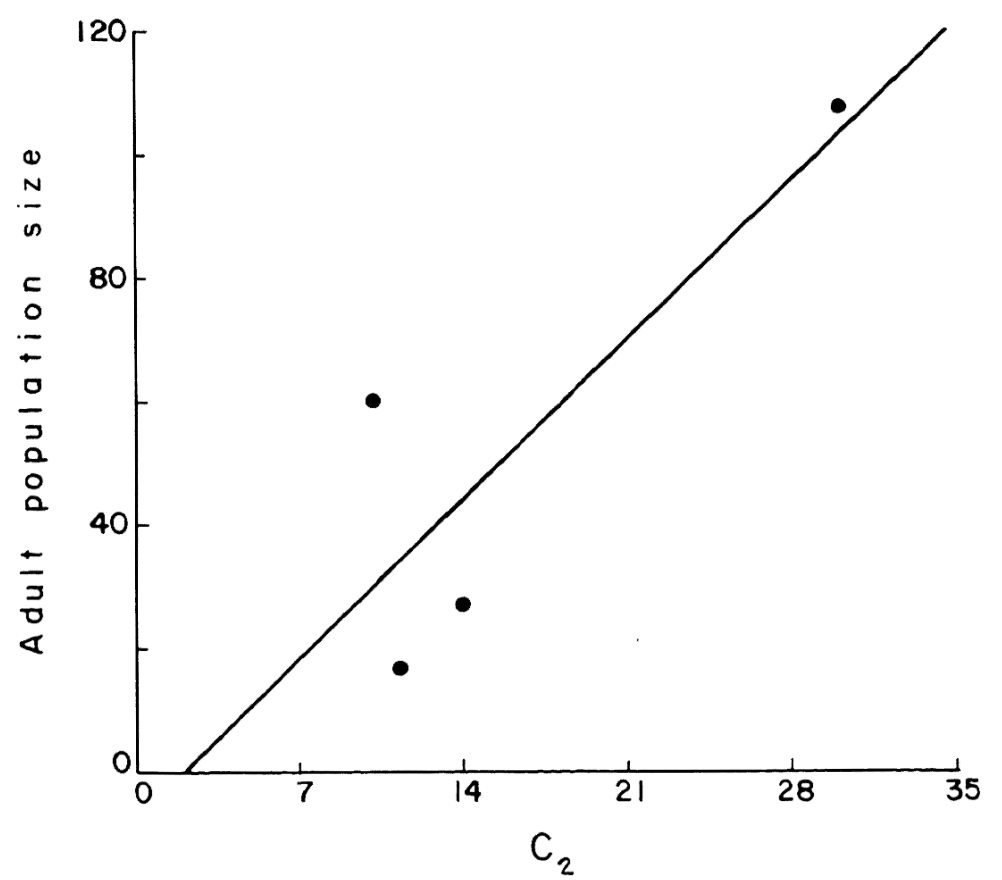

Fig. 2.-Population size as a function of euchresis. Population size, estimated by Hirose et al. (1980), represents adult population numbers in the area studied. The euchresis index was calculated using the equations in the Appendix.

population size (see above). A plot of the number of adults versus $C_{2}$ appears in figure 2. Unfortunately, there are too few points, and although the relationship is consistent with the theoretical predictions, it is not statistically significant.

\section{DISCUSSION}

The central point of this work is that the distribution of consumers over their resources has a profound influence on population size. This point was clearly stated by Andrewartha and Birch (1954) and later discussed in the context of stability of population equilibrium by Roff (1974) and more formally by Hassell and May (1974), Hassell (1978), and others. The theoretical development in the present work leads to the concept of euchresis, to the proposal of indexes to measure it, and to the hypothesis of a positive relationship between euchresis and population equilibrium.

One of the consequences of the theory is a new definition of carrying capacity, $K$, namely, the equilibrium population size given total euchresis. This definition is sensible only if one assumes that at least some individuals in the population have the behavioral or genetic flexibility required to alter their current use of elements (M. Rausher, pers. comm.). If a population with partial euchresis lacks such flexibility, carrying capacity will never be attained in ecological time, and therefore the definition may not be particularly useful. Notice that in this definition, 
potentially suitable, but not used, resources are not considered. Carrying capacity is defined in terms of those elements that are actually being used by members of the population. The addition of new elements, changes in the suitability of older ones (e.g., by increased digestive efficiency), or increased fecundity alter $K$. Behavioral, physiographical, or other changes altering only the pattern of use should produce changes in euchresis and thus, by hypothesis, in population size, without differences in $K$.

This leads to the question of how euchresis evolves. The evolution of euchresis must be considered from the point of view of optimal oviposition behavior, that is, individual selection. Models of this (Soberón 1982) suggested that, in general, individual selection for optimal oviposition tends to increase population euchresis. Crucial to this conclusion, however, is the requirement of a correct assessment of suitability, which as we saw in the introduction may be quite difficult for the exploiters.

There are two advantages of defining $K$ in terms of euchresis. First, it makes a clear distinction between the concepts of carrying capacity and equilibrium population. Usually, the equilibrium and $K$ are regarded as different things only when the action of natural enemies is considered (i.e., the $q$ of Beddington et al. 1978). When a population is assumed to be free of enemies, $K$ is often equated with equilibrium size (Slobodkin 1962; Pianka 1974; Southwood 1976; Begon and Mortimer 1981; see, however, Dempster and Pollard 1981; Pollard 1981). The distinction between the two concepts is interesting because an equilibrium population below its carrying capacity (i.e., a population with partial euchresis) is underutilizing at least part of its "available suitability," and this wastage may be of considerable importance. Competitors may take advantage of underutilized resources, and coexistence will be promoted. In equilibrium populations with partial euchresis, both intra- and interspecific competition would be difficult to detect by being restricted to a few elements (see also Shorrocks et al. 1979; Atkinson and Shorrocks 1981; Hanski 1981). Moreover, if partial euchresis were a common occurrence among herbivores, this would add to our understanding of "why the world is green" (Hairston et al. 1960; Ehrlich and Birch 1967). Current explanations for this include the action of natural enemies to keep herbivore populations well below their carrying capacities (Hassell 1978; Lawton and McNeill 1979; Lawton and Strong 1981) and the low food quality of much of what appears green to us (Sinclair 1975; White 1978). Imperfect euchresis implies that available and high-quality food is not being fully used.

The second advantage in using euchresis to define $K$ is that it provides a method for estimating its size. This method is different from the two traditional ones of using the maximum population size recorded in a place (Beddington et al. 1978) or the biomass of some important resource (Dempster and Pollard 1981). From the point of view of the present work, the former method can be misleading because if the maximum number is due to an outbreak, then clearly it will be an overestimate of $K$. On the other hand, if an average is taken of population size over several generations, it is the equilibrium size that has been estimated and not necessarily $K$ as defined here.

The latter method ( $K$ as the biomass of some resource), exemplified by Demp- 
ster and Pollard (1981), presents several problems. In the first place, it ignores the fact that, even according to some traditional definitions, the carrying capacity is set not only by availability of resources but also by fecundity and DI mortality. More relevant to the present discussion are the failure to recognize the difference between availability and suitability and the importance of heterogeneous suitabilities. Dempster and Pollard (1981) focused on an important problem by attempting to estimate $K$ from measures of resources; however, the estimates of resource biomass they proposed can be, at best, only proportional to $K$, and even then only by assuming that total suitability is equal to biomass. The coefficient of proportionality, however, cannot be specified without a model of the exploitation of the environment. The theory of euchresis proposes $C_{\max }$ as such a coefficient.

Dempster and Pollard (1981) complained about the scarcity of field measurements of $K$ and blamed the acceptance of the "equilibrium model" (the view that $n^{*}=K$ ) for this. The enormous complexity of the task, rather than the acceptance of the "equilibrium model," more readily explains the lack of studies of carrying capacity. Extensive life-table studies, over several kinds of resources and densities of exploitation, are required to quantify suitability, and these should be carried out in parallel with observations of the patterns of use. Such comprehensive studies are rare in the literature. I maintain, however, that such studies are needed if we are to understand more fully the dynamics of insect populations.

The theory of euchresis can be extended to include predator-prey or parasitoidhost interactions. Such development is difficult because of the complexity of the mathematical models involved. Reversals of the relation between euchresis and $n^{*}$ should be expected because a totally euchretic parasitoid would deplete its own food supplies more fully than a partially euchretic one (Soberón 1982). Euchresis theory may also provide a natural framework for the discussion of plantherbivore-parasitoid interactions (Lawton and McNeill 1979; Price et al. 1980). This will be discussed in a future paper.

\section{SUMMARY}

Carrying capacity cannot, in general, be proportional to the availability of resources. Rather, a perhaps complex relation between the patterns of use and the suitability of resources should determine the value of the carrying capacity of an animal population. The measurements both of use patterns and of suitability patterns are eased by restricting them to certain insect species that are monophagous as individuals. Mathematical modeling leads to a new concept, euchresis, defined as the degree of association between the pattern of the use of resources and the corresponding pattern of suitability, to the proposal of indexes to measure this, and to the hypothesis that population size and euchresis should be positively correlated whenever the resources are not dynamically coupled to the exploiters. Available data support the hypothesis.

\section{ACKNOWLEDGMENTS}

This work is part of my doctoral thesis for the Imperial College. I would like to thank my supervisor, M. P. Hassell, and M. Crawley for their patient and careful 
reading of the manuscript and for their advice and encouragement. M. Rausher, D. Piñero, R. Dirzo, and J. Llorente also read and commented on the manuscript. My wife Tita and C. Martinez del Rio contributed in no small part to the completion of this work, and the anonymous reviewers made several useful suggestions. To all of them, many thanks.

\section{APPENDIX}

The maximization of $n^{*}$ is outlined here, and the formulas for calculating $C_{\text {fit } 2}$ and $C_{\text {fit } 1}$ are presented. (Details of the procedure may be found in Soberón 1982.)

The equilibrium $n^{*}$ is implicitly defined by

$$
\Phi=\sum_{i} u_{i} \phi_{i}\left(u_{i} F n^{*}\right)=1 / F .
$$

Clearly, $n^{*}$ is a function of the $u_{i}$ 's. To maximize $n^{*}$ subject to $\Sigma_{i} u_{i}=1$, the method of the Lagrange multipliers was used. Let $\psi=\Sigma_{i} u_{i}$ and $g=n^{*}+\lambda \psi$, where $\lambda$ is the indeterminate Lagrange multiplier. The differential of $g$ is

$$
d g=d n^{*}+\lambda d \psi .
$$

By implicit differentiation of $n^{*}$ one obtains

$$
d g=\sum_{j}\left[\frac{-\left(u_{j} \partial \phi_{j} / \partial u_{j}+\phi_{j}\right)}{\sum_{i} u_{i} \partial \phi_{i} / \partial n^{* k}}+\lambda\right] d u_{j} .
$$

At a critical point all the elements of the sum vanish, which leads to the system

$$
u_{i}\left(\partial \phi_{i} / \partial u_{i}\right)+\phi_{i}=\lambda \sum_{j} u_{j}\left(\partial \phi_{j} / \partial n^{*}\right),
$$

and rearranging and summing over all $i$ yields an expression for $\lambda$. Substituting in equation (A3) finally produces a set of equations for the optimal fractional uses $\left(u_{i}\right)$ :

$$
u_{i}=\frac{\left[1+\sum_{j} \phi_{j}\left(\partial \phi_{j} / \partial u_{j}\right)^{-1}\right]\left(\partial \phi_{i} / \partial u_{i}\right)^{-1}}{\sum_{j}\left(\partial \phi_{j} / \partial u_{j}\right)^{-1}}-\phi_{i}\left(\partial \phi_{i} / \partial u_{i}\right)^{-1} .
$$

Equations (A4) have to be solved for each particular form of the survival functions $\phi_{i}$. In particular, equation (5) reduces (A4) to $u_{i}=s_{i}$ for all $i$.

Application of equations (A4) to the density-independent model of equation (10) gives, after a lot of tedious algebra, an expression for the carrying capacity and the euchresis. Let $\xi$ and $\gamma$ be defined as follows:

$$
\begin{gathered}
\xi=\frac{\sum_{j} s_{j} d_{j}}{\sum_{j} s_{j} / d_{j}}-\frac{1}{\left(\sum_{j} s_{j} / d_{j}\right)^{2}}, \\
\gamma=\left\{\left[(F D)^{2} \xi+\left(F D / \sum_{j} s_{j} / d_{j}-1\right)^{2}\right]^{1 / 2}+\left(F D / \sum_{j} s_{j} / d_{j}-1\right)\right\}^{-1} .
\end{gathered}
$$


Then,

$$
\begin{gathered}
C_{\max 2}=\frac{\left[F D\left(\gamma F D \xi+1 / \sum_{j} s_{j} / d_{j}\right)-1\right] \sum_{j} s_{j} / d_{j}}{(F D \gamma)^{2} \xi+1}, \\
K=\frac{S}{F^{2} D} C_{\max 2}, \\
C_{2}=\frac{F D \sum_{j} u_{j} d_{j}-1}{\sum_{j} u_{j}^{2} d_{j} / s_{j}}, \\
C_{\mathrm{fit} 2}=C_{2} / C_{\max 2}, \\
u_{i \text { opt }}=\frac{s_{i}}{d_{i} \sum_{j} s_{j} / d_{j}}\left[F D \gamma\left(d_{i}-\frac{1}{\sum_{j} s_{j} / d_{j}}\right)+1\right] .
\end{gathered}
$$

The corresponding values for equation (9) are

$$
\begin{aligned}
& \xi=\frac{1}{\sum_{j} s_{j} / d_{j}^{2}}-\left(\frac{\sum_{j} s_{j} / d_{j}}{\sum_{j} s_{j} / d_{j}^{2}}\right)^{2} \\
& \gamma=\left\{\left[(F D)^{2} \xi+\left(\frac{F D \sum_{j} s_{j} / d_{j}}{\sum_{j} s_{i j} / d_{j}^{2}}-1\right)^{2}\right]^{1 / 2}+\left(\frac{F D \sum_{j} s_{j} / d_{j}}{\sum_{j} s_{j} / d_{j}^{2}}-1\right)\right\}^{-1}, \\
& C_{\max 1}=\frac{\left[F D\left(\gamma F D \xi+\sum_{j} s_{j} / d_{j} / \sum_{j} s_{j} / d_{j}^{2}\right)-1\right] \sum_{j} s_{j} / d_{j}^{2},}{(F D \gamma)^{2} \xi+1}, \\
& K=\frac{S}{(F D)^{2}} C_{\max 1}, \\
& C_{1}=\left(F D \sum_{j} u_{j} d_{j}-1\right) /\left(\sum_{j} u_{j}^{2} d_{j}^{2} / s_{j}\right), \\
& C_{\text {fit } 1}=C_{1} / C_{\max 1} \text {, } \\
& u_{i \mathrm{opt}}=\frac{s_{i}}{d_{i}^{2} \sum_{j} s_{j} / d_{j}^{2}}\left[F D \gamma\left(d_{i}-\frac{\sum_{j} s_{j} / d_{j}}{\sum_{j} s_{j} / d_{j}^{2}}\right)+1\right] \text {. }
\end{aligned}
$$




\section{LITERATURE CITED}

Anderson, R. M. 1979. The influence of parasitic infection on the dynamics of host population growth. Pages 245-281 in R. M. Anderson, B. D. Turner, and L. R. Taylor, eds. Population dynamics. Blackwell, Oxford.

Andrewartha, H. G., and L. C. Birch. 1954. The distribution and abundance of animals. University of Chicago Press, Chicago.

Atkinson, W. D., and B. Shorrocks. 1981. Competition on a divided and emphemeral resource: a simulation model. J. Anim. Ecol. 50:461-471.

Atsatt, P. R. 1981. Ant-dependent food plant selection by the mistletoe butterfly Ogyris amaryllis (Lycaenidae). Oecologia (Berl.) 48:60-63.

Atsatt, P. R., and D. J. O'Dowd. 1976. Plant defense guilds. Science (Wash., D.C.) 193:24-29.

Beddington, J. R., C. A. Free, and J. H. Lawton. 1978. Characteristics of successful natural enemies in models of biological control of insect pests. Nature (Lond.) 273:513-519.

Begon, M., and M. Mortimer. 1981. Population ecology: a unified study of animals and plants. Blackwell, Oxford.

Bellows, T. S. 1981. The descriptive properties of some models for density dependence. J. Anim. Ecol. 50:139-156.

Chew, F. S. 1975. Coevolution of pierid butterflies and their cruciferous food plants. I. The relative quality of available resources. Oecologia (Berl.) 20:117-127.

1977. Coevolution of pierid butterflies and their cruciferous food plants. II. The distribution of eggs on potential food plants. Evolution 31:568-579.

Clark, L. R., P. W. Geier, R. D. Hughes, and R. F. Morris. 1967. The ecology of insect populations in theory and practice. Methuen, London.

Copp, N. H., and D. Davenport. 1978. Agraulis and Passiflora control of specificity. Biol. Bull. (Woods Hole) 155:98-112.

Courtney, S. P. 1981. Coevolution of pierid butterflies and their cruciferous food plants. III. Anthocharis cardamines (L.) survival, development and oviposition on different host plants. Oecologia (Berl.) 51:91-96.

Crawley, M. 1983. Herbivory: the dynamics of animal-plant interactions. Blackwell, Oxford.

Dempster, J. P., and E. Pollard. 1981. Fluctuations in resource availability and insect populations. Oecologia (Berl.) 50:412-416.

Dethier, V. G. 1959. Food-plant distribution and density and larval dispersal as factors affecting insect populations. Can. Entomol. 91:581-596.

Dirzo, R. 1980. Experimental studies on slug-plant interactions. I. The acceptability of thirty plant species to the slug Agriolimax caruanae. J. Ecol. 68:981-998.

- 1984. Herbivory: a phytocentric overview. Pages 141-165 in R. Dirzo and J. Sarukhan, eds. Perspectives in plant population ecology. Sinauer, Sunderland, Mass.

Dolinger, P. M., P. R. Ehrlich, W. L. Fitch, and D. E. Breedlove. 1973. Alkaloid and predation patterns in Colorado lupine populations. Oecologia (Berl.) 13:191-204.

Ehrlich, P. R., and L. C. Birch. 1967. "The balance of nature" and "population control." Am. Nat. 101:97-107.

Feeny, P. 1970. Seasonal changes in oak leaf tannins and nutrients as a cause of spring feeding by winter moth caterpillars. Ecology 51:565-581.

Gilbert, L. E. 1975. Ecological consequences of a coevolved mutualism between butterflies and plants. Pages 210-240 in L. E. Gilbert and P. H. Raven, eds. Coevolution of animals and plants. University of Texas Press, Austin.

- 1977. The role of coevolution in the organization of ecosystems. Pages 399-413 in V. Labyrie, ed. Comportment des insects et milieu trophique. C.N.R.S., Paris.

Hairston, N. G., F. E. Smith, and L. B. Slobodkin. 1960. Community structure, population control, and competition. Am. Nat. 94:421-425.

Hanski, I. 1981. Coexistence of competitors in patchy environments with and without predation. Oikos 37:306-312.

Harcourt, D. F. 1966. Major factors in survival of the immature stages of Pieris rapae (L.). Can. Entomol. 98:653-662. 
Hassell, M. P. 1978. The dynamics of arthropod predator-prey systems. Princeton University Press, Princeton, N.J.

Hassell, M. P., and R. M. May. 1974. Aggregation in predators and insect parasites and its effects on stability. J. Anim. Ecol. 43:567-594.

Haukioja, E. 1980. On the role of plant defences in the fluctuations of herbivore populations. Oikos 35:202-213.

Haukioja, E., and P. Niemela. 1977. Retarded growth of a geometrid larva after mechanical damage to leaves of its host tree. Ann. Zool. Fenn. 14:48-52.

Haukioja, E., P. Niemela, and L. Iso-livari. 1978a. Birch leaves as a resource for herbivores. I. Variation in the suitability of leaves. Rep. Kevo Subarct. Res. Stn. 14:5-12.

$1978 b$. Birch leaves as a resource for herbivores. II. Diurnal variation in the suitability of leaves for Oporinia autumnata and Dineura virididorsata. Rep. Kevo Subarct. Res. Stn. 14:21-24.

Hinton, H. E. 1951. Myrmecophilous Lycaenidae and other Lepidoptera: a summary. Trans. South Lond. Entomol. Nat. Hist. Soc. 1949-1950:111-175.

Hirose, Y., Y. Suzuki, M. Takagi, K. Hiehata, H. Yamasaki, H. Kimoto, M. Yamanaka, M. Iga, and - K. Yamaguchi. 1980. Population dynamics of the citrus swallowtail Papilio xuthus Linné (Lepidoptera: Papilionidae): mechanisms stabilizing its numbers. Res. Popul. Ecol. (Kyoto) 21:260-285.

Hocking, B. 1975. Ant-plant mutualism: evolution and energy. Pages 78-90 in L. E. Gilbert and P. H. Raven, eds. Coevolution of animals and plants. University of Texas Press, Austin.

Holdren, C. E., and P. R. Ehrlich. 1982. Ecological determinants of food plant choice in the checkerspot butterfly Euphydryas editha in Colorado. Oecologia (Berl.) 52:417-423.

Hurlbert, S. H. 1978. The measurement of niche overlap and some relatives. Ecology 59(1):67-77.

Janzen, D. 1966. Coevolution of mutualism between ants and acacias in Central America. Evolution 20:249-275.

Kaufmann, T. 1965. Biological studies on some Bavarian Acridoidea (Orthoptera) with special reference to their feeding habits. Ann. Entomol. Soc. Am. 58(6):791-801.

King, C. E. 1971. Resource specialization and equilibrium population size in patchy environments. Proc. Natl. Acad. Sci. USA 68:2634-2637.

Lack, D. 1954. The natural regulation of animal numbers. Clarendon Press, Oxford.

Lawton, J. H., and S. McNeill. 1979. Between the devil and the deep blue sea: on the problem of being a herbivore. Pages 223-244 in R. M. Anderson, B. D. Turner, and L. R. Taylor, eds. Population dynamics. Blackwell, Oxford.

Lawton, J. H., and D. R. Strong. 1981. Community patterns and competition in folivorous insects. Am. Nat. 118:317-338.

MacArthur, R. 1968. The theory of the niche. Pages 159-176 in R. C. Lewontin, ed. Population biology and evolution. Syracuse University Press, Syracuse, N.Y.

MacArthur, R., and R. Levins. 1964. Competition, habitat selection and character displacement in a patchy environment. Proc. Natl. Acad. Sci. USA 51:1207-1210.

Mattson, W. J., Jr. 1980. Herbivory in relation to plant nitrogen content. Annu. Rev. Ecol. Syst. 11:119-161.

May, R. M., M. P. Hassell, R. M. Anderson, and D. W. Tonkyn. 1981. Density dependence in hostparasitoid models. J. Anim. Ecol. 50:855-865.

Maynard Smith, J. 1968. Mathematical ideas in biology. Cambridge University Press, Cambridge.

McNaughton, S. J., and L. L. Wolf. 1979. General ecology. 2d ed. Holt, Rinehart \& Winston, New York.

Mitchell, R. 1981. Insect behaviour, resource exploitation and fitness. Annu. Rev. Entomol. 26:373396.

Petraitis, P. S. 1979. Likelihood measures of niche breadth and overlap. Ecology 60(4):703-710.

- 1981. Algebraic and graphical relationships among niche breadth measures. Ecology 62(3):545-548.

Pianka, E. R. 1974. Evolutionary ecology. Harper \& Row, New York.

Pollard, E. 1981. Resource-limited and equilibrium models of populations. Oecologia (Berl.) 49: 371-378. 
Price, P. W., C. E. Bouton, P. Gross, B. A. McPheron, J. N. Thompson, and A. E. Weis. 1980. Interactions among three trophic levels: influence of plants on interactions between insect herbivores and natural enemies. Annu. Rev. Ecol. Syst. 11:41-65.

Prokopy, R. J. 1972. Evidence for a marking pheromone deterring repeated oviposition in apple maggot flies. Environ. Entomol. 1:326-332.

Rausher, M. D. 1979a. Larval habitat suitability and oviposition preference in three related butterflies. Ecology 60(3):503-511.

1979b. Egg recognition: its advantage to a butterfly. Anim. Behav. 27:1034-1040.

1980. Host abundance, juvenile survival and oviposition preference in Battus philenor. Evolution 34(2):342-355.

Ricklefs, R. E., and M. Lau. 1980. Bias and dispersion of overlap indices: results of some Monte Carlo simulations. Ecology 61(5):1019-1024.

Roff, D. A. 1974. Spatial heterogeneity and the persistence of populations. Oecologia (Berl.) 15: 245-258.

Rothschild, M., and L. M. Schoonhoven. 1977. Assessment of egg load of Pieris brassicae (Lepidoptera: Pieridae). Nature (Lond.) 266:352-355.

Roughgarden, J. 1974. Population dynamics in a spatially varying environment: how population size "tracks" spatial variation in carrying capacity. Am. Nat. 108:649-664.

Scriber, J. M. 1979. The effects of sequentially switching food plants upon biomass and nitrogen utilization by polyphagous and stenophagous Papilio larvae. Entomol. Exp. Appl. 25:203215.

Shapiro, A. H. 1981. Egg-mimics of Streptanthus (Cruciferae) deter oviposition by Pieris sisymbrii (Lepidoptera: Pieridae). Oecologia (Berl.) 48:142-143.

Shorrocks, B., W. Atkinson, and P. Charlesworth. 1979. Competition on a divided and ephemeral resource. J. Anim. Ecol. 48:899-908.

Sinclair, A. R. E. 1975. The resource limitation of trophic levels in tropical grasslands ecosystems. J. Anim. Ecol. 44:497-520.

Singer, M. C. 1972 . Complex components of habitat suitability within a butterfly colony. Science (Wash., D.C.) 176:75-77.

Slobodkin, L. B. 1962. Growth and regulation of animal populations. Holt, Rinehart \& Winston, New York.

Smiley, J. 1978. Plant chemistry and the evolution of host specificity: new evidence from Heliconius and Passiflora. Science (Wash., D.C.) 201:745-747.

Smith, E. P. 1982. Niche breadth, resource availability and inference. Ecology 63(6):1675-1681.

Soberón, J. 1982. Insect population dynamics in heterogeneous environments. Ph.D. diss. Imperial College, London.

Solbrig, O. T., and D. Solbrig. 1979. Introduction to population biology and evolution. AddisonWesley, Reading, Mass.

Southwood, T. R. E. 1976. Bionomic strategies and population parameters. Pages $26-48$ in R. M. May, ed. Theoretical ecology: principles and applications. Saunders, Philadelphia.

Strebel, D. E. 1980. Logistic growth in the presence of non-white environmental noise. J. Theor. Biol. 85:713-718.

Vinson, S. 1976. Host selection by insect parasitoids. Annu. Rev. Entomol. 21:109-133.

Waage, J. K., and M. P. Hassell. 1982. Parasitoids as biological control agents-a fundamental approach. Parasitology 84:241-268.

Wangersky, P. J., and W. J. Cunningham. 1957. Time lag in predator-prey population models. Ecology 38:136-139.

White, T. C. R. 1978. The importance of relative shortage of food in animal ecology. Oecologia (Berl.) 33:71-86.

Wiens, J. A. 1976. Population responses to patchy environments. Annu. Rev. Ecol. Syst. 7:81-120.

Wiklund, C. 1981. Generalist vs. specialist oviposition: oviposition preferences. Oikos 31:169-183.

Wiklund, C., and K. Ahrberg. 1978. Host plants, nectar source plants and habitat suitability of males and females of Anthocharis cardamines (Lepidoptera). Oikos 31:169-183.

Williams, K. S., and L. E. Gilbert. 1981. Insects as selective agents on plant vegetative morphology: egg mimicry reduces egg laying by butterflies. Science (Wash., D.C.) 212:467-469. 